\title{
THE SECOND NORMAL BORDISM GROUP OF A SPACE PAIR
}

\author{
GUI-SONG LI
}

(Communicated by Frederick R. Cohen)

\begin{abstract}
We determine the second normal bordism groups of space pairs in terms of their homology groups except for certain group extension problems.
\end{abstract}

Given a stable vector bundle $\phi$ over a pair of topological spaces $(X, A)$ and an integer $n \geq 0$, we can define an abelian group $\Omega_{n}(X, A ; \phi)$ called the $n t h$ normal bordism group of $(X, A)$ with coefficients in $\phi$ (see $[1,3,10])$. These bordism groups are of relevance in the immersion and embedding theory. For example, in certain cases, the enumeration problem of differential immersions and embeddings of manifolds into manifolds may be converted into the problem of enumerating elements in certain normal bordism groups by $[1,3,10,11]$.

Explicit computations of normal bordism groups are extremely difficult in general. In [3] Koschorke developed an exact sequence that enables us to determine $\Omega_{n}(X ; \phi)$ for $n \leq 2$ except for certain group extension problems. These extension problems have been solved partially by a recent work of $\mathrm{Li}$ [5]. The Koschorke sequence is the basis of almost all the known work on singularity approaches to immersions (see, e.g., [2-8]). However, to study embeddings in the normal bordism framework, one has to use the normal bordism group of a certain space pair $(X, A)$ where $A$ is nonempty. The bordism group $\Omega_{n}(X, A ; \phi)$ is simple when $n \leq 1$ because in these cases, it is easy to see that if $X$ and $A$ are path-connected then $\Omega_{n}(X, A ; \phi)$ is canonically isomorphic to $H_{n}\left(X, A ; \mathbf{Z}_{\phi}\right)$ where $\mathbf{Z}_{\phi}$ is the integer coefficient system twisted by $w_{1}(\phi)$. This has been applied in $[6,7]$ to the enumeration problem of embeddings of $n$-manifolds into $(2 n+1)$ - and $2 n$-manifolds.

In this paper we determine the bordism group $\Omega_{2}(X, A ; \phi)$ in terms of homology groups of $(X, A)$ except for certain group extension problems. Our result can be used to enumerate embeddings of $n$-manifolds into $(2 n-1)$ manifolds. To do this, one needs explicit computations of homology groups of a certain very complicated space pair, which is not the subject of this paper.

Throughout this paper we shall follow Salomonsen's definition of normal bordism groups in [10]. So $\Omega_{n}(X, A ; \phi)$ is the bordism group of triples of the form $(M, f, \bar{f})$ where $M$ is a compact smooth $n$-manifold, $f:(M, \partial M) \rightarrow$

Received by the editors August 12, 1991.

1991 Mathematics Subject Classification. Primary 57R90; Secondary 57R40.

Key words and phrases. Relative normal bordism groups, Koschorke sequences, singularity sets. 
$(X, A)$ is a map, and $\bar{f}: T M \rightarrow f^{*} \phi$ is a stable bundle isomorphism. Salomonsen's normal bordism group is canonically isomorphic to that of Dax and Koschorke in $[1,3]$ but uses the opposite sign convention for the stable vector bundle $\phi$. The reader is referred to $[1,10]$ for the basic properties of normal bordism groups.

We now come to the main result of this paper.

Theorem. Let $X$ and $A$ be homotopically equivalent to connected CWcomplexes with finite skeletons in all dimensions, $X \supset A \neq \varnothing$, and let $\phi$ be a stable vector bundle over $X$. Then $\Omega_{2}(X, A ; \phi)$ is an extension of $H_{2}\left(X, A ; \mathbf{Z}_{\phi}\right)$ by Coker $\Phi$. Here $\Phi: H_{3}\left(X, A, \mathbf{Z}_{\phi}\right) \rightarrow H_{1}\left(X, A ; \mathbf{Z}_{2}\right)$ is defined by $\Phi(x)=\bar{w}_{2}(\phi) \cap \rho(x)$ and $\rho$ is the modulo two reduction.

It seems possible to prove the theorem along the lines previously used by Koschorke [3] in developing his exact sequence. One needs first to define a relative version of Koschorke's fine bordism groups and then check the validity of all necessary constructions and proofs in [3] for manifolds with boundary. Here, instead of making these tedious checks, we derive our theorem directly from the "absolute" Koschorke sequence using certain geometric considerations.

Let $\bar{\Omega}_{n}(X, A ; \phi)$ be the bordism group of triples of the form $(M, f, \alpha)$ where $M$ is a compact smooth $n$-manifold, $f:(M, \partial M) \rightarrow(X, A)$ is a map, and $\alpha: \xi_{T M} \cong \xi_{f^{*} \phi}$ is an isomorphism between orientation line bundles. Then there is a natural forgetful homomorphism

$$
\mu: \Omega_{n}(X, A ; \phi) \rightarrow \bar{\Omega}_{n}(X, A ; \phi)
$$

defined by forgetting about a stable bundle isomorphism and retaining only the underlying orientation information. Moreover, if $n \leq 3$ then there is a natural isomorphism

$$
\theta: \bar{\Omega}_{n}(X, A ; \phi) \cong H_{n}\left(X, A ; \mathbf{Z}_{\phi}\right)
$$

defined by $\theta(x)=f_{*}([M])$ where $x$ is represented by a triple $(M, f, \alpha)$ and $[M] \in H_{n}\left(M, \partial M ; \mathbf{Z}_{T M}\right)$ is the fundamental class of $M$. The special case where $A$ is empty was proved by Olk [9]. The general case can be proved by comparing the long exact sequence of homology groups of $(X, A)$ with the corresponding exact sequence involving $\bar{\Omega}_{n}$.

We now consider the following diagram:

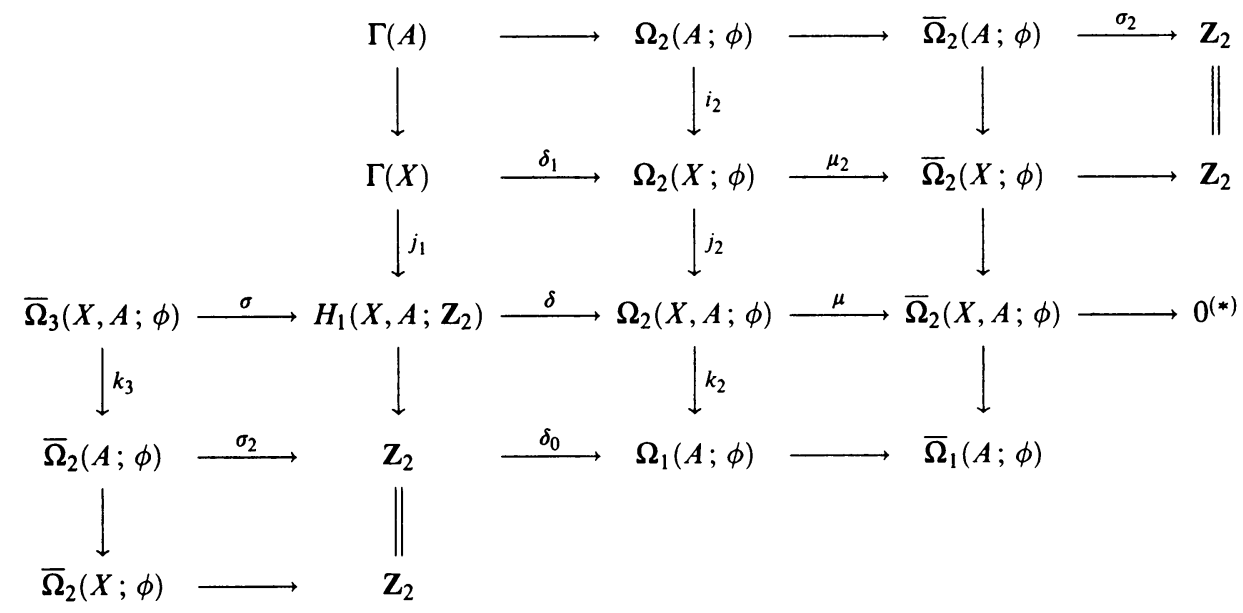


where $\Gamma(X)$ denotes a certain normal bordism group of $X \times B O(2)$. The vertical sequences above are long exact sequences of normal bordism groups of space pairs and the horizontal sequences, except the one marked $(*)$, are exact Koschorke sequences given in Theorem 9.3 of [3] (note, however, slightly different notations were used there). The assumption on $X$ and $A$ ensures the existence of these Koschorke sequences.

The above homomorphism $\delta$ is defined as follows: Let $x \in H_{1}\left(X, A ; \mathbf{Z}_{2}\right)$. Since $j_{1}$ is surjective, there is an element $y \in \Gamma(X)$ so that $j_{1}(y)=x$. We define $\delta(x)=j_{2} \delta_{1}(y)$. The homomorphism $\sigma$ is defined by $\sigma=\Phi \circ \theta$. It is not difficult to see that these homomorphisms are well defined and make the above diagram commute.

In the remainder of the paper we shall prove the exactness of the horizontal sequence marked $(*)$ in the diagram above, which by virtue of our previous discussions, immediately leads to the conclusions of our main theorem.

For our purpose we shall need a geometric interpretation of the homomorphism $\sigma$. Let $x \in \bar{\Omega}_{3}(X, A ; \phi)$ be represented by a map $F:(W, \partial W) \rightarrow$ $(X, A)$ and an isomorphism $\alpha: \xi_{T W} \cong \xi_{F^{*} \phi}$ where $W$ is a compact smooth 3-manifold. Then $T W-F^{*} \phi$ is stably equivalent to a 2-dimensional vector bundle $\psi$ over $W$. Since $\psi$ is orientable, it is trivial if and only if there is a nonzero section of $\psi$. Take a nondegenerate section $u$ of $\psi$ over $W$. Then the zero-point set $S$ of $u$, which is the singularity set for finding a stable trivialization of $T W-F^{*} \phi$, is a compact smooth 1 -manifold with $\partial S=S \cap \partial W$. We claim that $\sigma(x)$ is represented by $S$ in the sense that $\sigma(x)=F_{*}\left([S]_{2}\right)$ where $[S]_{2} \in H_{1}\left(W, \partial W ; \mathbf{Z}_{2}\right)$ is determined by $S$. To prove this one may note that $[S]_{2}=F^{*} \bar{w}_{2}(\phi) \cap \rho([W])$ because it can be seen from Chapter II of [1] that $[S]_{2}$ is the Poincare dual of $w_{2}(\psi)=w_{2}\left(T W-F^{*} \phi\right)=F^{*} \bar{w}_{2}(\phi)$. The assertion now follows by the naturality of the cap product.

We now prove the exactness of the sequence $(*)$, which can be viewed as a relative version of the Koschorke sequence.

(1) Let $x \in \bar{\Omega}_{2}(X, A ; \phi)$ be represented by a map $f:(M, \partial M) \rightarrow(X, A)$ and an isomorphism $\alpha: \xi_{T M} \cong \xi_{f^{*} \phi}$ where $M$ is a compact smooth 2-manifold. Since $T M-f^{*} \phi$ is orientable, it is stably trivial restricted over $M$ with a point $p \in M-\partial M$ removed. One may assume that there is an open disk $D \subset M-\partial M$ around $p$ so that $f(\bar{D}) \subset A$. Since the triple $(M, f, \alpha)$ restricted to $M-D$ also represents $x$, it follows immediately that $x \in \operatorname{Im} \mu$. This proves that $\mu$ is surjective.

(2) To check the exactness at $\Omega_{2}(X, A ; \phi)$, note that obviously $\mu \circ \delta=0$. On the other hand, let $x \in \Omega_{2}(X, A ; \phi)$ satisfy $\mu(x)=0$. We claim that $k_{2}(x)=0$. Assuming this result for a moment, we can choose an $x^{\prime} \in \Omega_{2}(X ; \phi)$ with $j_{2}\left(x^{\prime}\right)=x$. An easy diagram chasing shows that there exist $y \in \Omega_{2}(A ; \phi)$ and $z \in \Gamma(X)$ so that $\mu_{2} i_{2}(y)=\mu_{2}\left(x^{\prime}\right)$ and $\delta_{1}(z)=x^{\prime}-i_{2}(y)$. Clearly, $j_{2} \delta_{1}(z)=j_{2}\left(x^{\prime}\right)=x$. This proves that $\operatorname{Ker} \mu \subseteq \operatorname{Im} \delta$ as desired.

To prove $k_{2}(x)=0$, let $x$ be represented by a map $f:(M, \partial M) \rightarrow(X, A)$ and a stable bundle isomorphism $\bar{f}: T M \rightarrow f^{*} \phi$ where $M$ is a compact smooth 2-manifold. The vanishing of $\mu(x)$ implies: (i) There is a compact smooth 2manifold $N$ and a map $g: N \rightarrow A$ so that $\partial N=\partial M$ and $\left.g\right|_{\partial N}=\left.f\right|_{\partial M}$; (ii) There is a compact smooth 3-manifold $W$ and a map $F: W \rightarrow X$ so that $\partial W=M \cup N,\left.F\right|_{\partial W}=f \cup g$, and $T W-f^{*} \phi$ is orientable. Let $u$ be the 
stable trivialization of $\left.\left(T W-F^{*} \phi\right)\right|_{M}$ determined by $\bar{f}$. Then the singularity set $S$ for extending $u$ to a stable trivialization of $T W-F^{*} \phi$ is a compact smooth 1-manifold. The boundary of $S$ then is the singularity set for extending $\left.u\right|_{\partial N}$ to a stable trivialization of $\left.\left(T W-F^{*} \phi\right)\right|_{N}$. Let $r \in \mathbf{Z}_{2}$ be the modulo 2 number of boundary points of $S$. Then $\delta_{0}(r)=k_{2}(x)$ by [3]. This implies that $k_{2}(x)=0$ since the number of points in $\partial S$ must be even.

(3) We now prove the exactness at $H_{1}\left(X, A ; \mathbf{Z}_{2}\right)$. Let $x \in \bar{\Omega}_{3}(X, A ; \phi)$ be represented by a map $F:(W, \partial W) \rightarrow(X, A)$ and an isomorphism $\alpha: \xi_{T W} \cong$ $\xi_{F^{*} \phi}$ where $W$ is a compact 3-manifold. Then $\sigma_{2} k_{3}(x)=0$ in the above diagram. This implies the existence of a stable trivialization $u$ of $T W-F^{*} \phi$ over $\partial W$. Such a trivialization will define an element $y \in \Omega_{2}(A ; \phi)$ that clearly satisfies $\mu_{2} i_{2}(y)=0$. We can then choose an element $z \in \Gamma(X)$ so that $\delta_{1}(z)=i_{2}(y)$. Since the triple $(W, F, \alpha)$ above also gives us a null-bordism of $\mu_{2} i_{2}(y)$ in $\bar{\Omega}_{2}(X ; \phi), z$ may be represented by the singularity set for extending $u$ to a stable trivialization of $T W-F^{*} \phi$ over $W$ by [3]. It follows by definition that $\sigma(x)=j_{1}(z)$ and that $\delta \sigma(x)=j_{2} \delta_{1}(z)=j_{2} i_{2}(y)=0$.

Conversely, given $z \in \Gamma(X)$ satisfying $j_{2} \delta_{1}(z)=0$, there exists $y \in$ $\Omega_{2}(A ; \phi)$ so that $i_{2}(y)=\delta_{1}(z)$. Let $\delta_{1}(z)$ be represented by a map $f: M \rightarrow A$ and a stable trivialization of $T M-f^{*} \phi$ where $M$ is a closed smooth 2manifold. Then there is a compact smooth 3-manifold $W$ with boundary $M$, a map $F: W \rightarrow X$ extending $f$, and an isomorphism $\alpha: \xi_{T W} \cong \xi_{F^{*} \phi}$ that restricts to the one induced by the stable trivialization of $T M-f^{*} \phi$ over $M$. This defines an element $x \in \bar{\Omega}_{3}(X, A ; \phi)$, which in view of discussions in the last paragraph clearly satisfies $\sigma(x)=j_{1}(z)$.

The proof of the theorem is now complete.

\section{REFERENCES}

1. J.-P. Dax, Étude homotopique des espaces de plongements, Ann. Sci. École Norm. Sup. (4) 6 (1972), 303-377.

2. U. Kaiser and B.-H. Li, Enumeration of immersions of m-manifolds in $(2 m-2)$-manifolds by the singularity method, Lecture Notes in Math., vol. 1350, Springer-Verlag, Berlin and New York, 1988, pp. 171-187.

3. U. Koschorke, Vector fields and other vector bundle morphisms-a singularity approach, Lecture Notes in Math., vol. 847, Springer-Verlag, Berlin and New York, 1981.

4. The singularity method and immersions of m-manifolds into manifolds of dimension $2 m-2,2 m-3,2 m-4$, Lecture Notes in Math., vol. 1350, Springer-Verlag, Berlin and New York, 1988, pp. 188-212.

5. B.-H. Li, On Koschorke's normal bordism sequence, Sci. Sinica (to appear).

6. __ Embeddings of n-manifolds into $2 n$-manifolds, preprint.

7. B.-H. Li and P. Zhang, On isotopy classification of embeddings of $n$-manifolds into $2 n$ manifolds, preprint.

8. G.-S. Li, On immersions in bordism classes, Math. Ann. 291 (1991), 373-382.

9. C. Olk, Immersionen von Mannigfaltigkeiten in Euklidische Räume, Dissertation, Siegen, 1980.

10. H. A. Salomonsen, Bordism and geometric dimension, Math. Scand. 32 (1973), 87-111.

11. On the existence and classification of differential embeddings in the metastable range, Mimeographed Notes, Aärhus Univ., 1973. 